\title{
Laparoscopic lumbar hernia repair with bone anchor fixation
}

\author{
Vanessa P. Ho · Gregory F. Dakin
}

Received: 10 April 2010/ Accepted: 3 September 2010/Published online: 29 October 2010

(C) Springer Science+Business Media, LLC 2010

\begin{abstract}
Introduction Lumbar hernias are rare defects of the posterolateral abdominal wall. Surgical repair of lumbar hernias is challenging because they are bounded inferiorly by the iliac bone, which makes adequate mesh fixation difficult. We demonstrate a method of a laparoscopic lumbar hernia repair utilizing bone anchor fixation at the inferior border.

Methods The patient is a 37-year-old male who had been in a motor vehicle collision and presented with a large left lumbar hernia and nonspecific abdominal pain. The patient had a Petit-type hernia that was bordered by the external oblique muscle, the latissimus dorsi, and the iliac crest. We opted to perform a laparoscopic mesh repair. Two Mitek GII QuickAnchor sutures were placed in the anterior superior iliac crest to provide inferior fixation of the mesh, with sufficient overlap of the mesh to prevent recurrence. The remainder of the mesh was fixed with standard laparoscopic tacks and sutures under good visualization to avoid damage of underlying structures.

Results The patient did well postoperatively and left the hospital on the first postoperative day. He has had no signs of recurrence at follow-up.
\end{abstract}

Presented at the 12th WCES, April 14-17, 2010, National Harbor, MD.

Electronic supplementary material The online version of this article (doi:10.1007/s00464-010-1400-8) contains supplementary material, which is available to authorized users.

V. P. Ho · G. F. Dakin $(\bowtie)$

Department of Surgery, NewYork-Presbyterian Hospital/Weill

Cornell Medical Center, Weill Cornell Medical College,

525 East 68 Street, New York, NY 10065, USA

e-mail: grd9006@med.cornell.edu
Conclusions The anatomic features of lumbar hernias create several challenges. A number of surgical approaches for lumbar hernia repair have been described, including laparoscopic and open methods as well as intraperitoneal and preperitoneal approaches and the use of flaps to cover the defects. However, limited fixation points for the mesh can lead to high recurrence rates. Bone anchors have been used in a variety of surgical disciplines, including orthopedics, plastic surgery, and gynecologic surgery, with low complication rates of bone pain or infection. We demonstrated a method that utilizes bone anchor fixation in a laparoscopic approach to overcome the challenge of inferior fixation. This securely repairs the hernia with good coverage of the defect while maintaining the benefits of the minimally invasive approach.

Disclosures Drs. Ho and Dakin have no conflicts of interest or financial ties to disclose. 